\title{
Three Years of Evaluation to Determine Reduction of Antibiotic Resistance in Gram-Negative Bacteria by the Saudi National Action Plan
}

This article was published in the following Dove Press journal:

Infection and Drug Resistance

\author{
Meshari Alabdullatif ${ }^{1,2}$ \\ Jihad Alrehaili' \\ 'Department of Pathology, College of \\ Medicine, Imam Mohammad Ibn Saud \\ Islamic University, Riyadh, Saudi Arabia; \\ ${ }^{2}$ Department of Microbiology, SmartLab, \\ National Health Laboratory Services, \\ Riyadh, Saudi Arabia
}

Background: Bacterial antibiotic resistance (AR) is a primary public health concern. In 2017, the Saudi National Action Plan (SNAP) implemented several strategies to overcome AR. Here, to better understand the effectiveness of that plan, we evaluated the rates of AR, extended-spectrum beta-lactamase (ESBL) positivity, and multi-drug resistance (MDR) among gram-negative bacteria in a private Saudi hospital.

Methods: This retrospective study included all patients with a confirmed diagnosis of gramnegative bacterial infection from January 2017 to December 2019. Identification of bacterial strains was performed using VITEK 2 ID-GNB cards, while AR, ESBL, and MDR were determined using AST-No. 12 cards, both used as recommended by the manufacturer. Cards were loaded into a VITEK 2 system for examination.

Results: A total of 4760 isolated gram-negative bacteria were collected. The most isolated organism was Escherichia coli, with $2585 / 4760$ (54.30\%) strains, and the least was Providencia stuartii, with 55/4760 (1.16\%) strains. A total of 1328/4760 (27.90\%) clinical isolates were ESBL-positive, and 851/4760 (17.88\%) possessed MDR. Escherichia coli was also the most frequently isolated as having ESBL activity and MDR, with 772/1328 (58.13\%) and 292/851 (34.31\%) isolates, respectively. Between 2017 and 2019, the rates of ESBL and MDR were significantly reduced $(p<0.05)$ for most bacteria, except for Salmonella species, which showed increased resistance to antibiotics.

Conclusion: Our findings revealed that the rates of AR, ESBL, and MDR reduced over time, which suggests the SNAP is effective at overcoming AR risk.

Keywords: antibiotic resistance, multi-drug resistance, gram-negative bacteria, VITEK, Saudi Arabi

\section{Introduction}

Antibiotic resistance (AR) poses a major risk for public health, ${ }^{1,2}$ particularly as widespread overuse of antibiotics throughout the past 80 years has led to increased rates of AR in bacteria and limited our antibiotic resources. ${ }^{3}$ AR bacteria have been reported to contribute to 700,000 deaths annually, a toll that is expected to rise to as many as 10 million deaths per year by 2050 if appropriate prevention and control measures are not followed. ${ }^{4,5}$ Thus, there is a great need to address the misuse and inappropriate prescription of antibiotics; these activities could contribute to the development of extended-spectrum beta-lactamase (ESBL) and multidrug-resistant (MDR) bacteria. ${ }^{6}$

The World Health Organization (WHO) has released a global action plan that highlights increasing consciousness of $\mathrm{AR}$ as a critical action to be undertaken
Correspondence: Meshari Alabdullatif Department of Pathology, College of Medicine, Imam Mohammad Ibn Saud Islamic University, Uthman Ibn Affan Road, Riyadh 13317-4233, Saudi Arabia

Tel +966 ||-258-28||

Fax +966 II-203-7I09

Email MIAlabdullatif@imamu.edu.sa
Infection and Drug Resistance 2020:13 3657-3667

3657

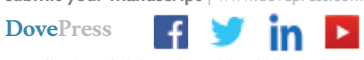

http://doi.org/10.2147/1DR.S265000 
worldwide. $^{7,8}$ In 2017, the WHO also issued the Saudi National Action Plan (SNAP), which was developed by the Ministry of Health to address the development of AR in bacteria and guarantee the continuity of successful treatment and prevention of infection through the provision of effective and safe medicines. ${ }^{9,10}$ Key strategies implemented in this plan are: enhancing consciousness and understanding of AR; supporting relevant knowledge and evidence bases through research and scientific meetings; reducing the incidence of infection through effective sanitation and hygiene; optimizing the use of antibiotic medicines, and increasing investment in new medicines, vaccines, and other interventions. $^{10,11}$

In this study, we investigated the effectiveness of these implemented strategies by retrospectively evaluating the $\mathrm{AR}, \mathrm{ESBL}$, and MDR rates of gram-negative bacteria over a three-year period in one of the biggest hospitals in Saudi Arabia.

\section{Materials and Methods Study Design}

This retrospective study was performed in a private 120bed hospital in Riyadh, Saudi Arabia, starting in January 2017 and continuing to December 2019. The study subjects comprised all patients with a confirmed diagnosis of gram-negative bacterial infection, specifically infection with Escherichia coli, Klebsiella pneumoniae, Pseudomonas aeruginosa, Proteus species, Acinetobacter species, Enterobacter cloacae, Citrobacter species, Salmonella species, or Providencia stuartii; other gramnegative bacteria were excluded from analysis. A total of 4760 gram-negative bacterial isolates were collected, identified, and evaluated for antibiotic susceptibility. These isolates were collected from the following departments: outpatient care $(2126 / 4760 ; 44.66 \%)$, emergency (1086/ $4760 ; 22.82 \%)$, intensive care unit $(638 / 4760 ; 13.4 \%)$, multi-service ward $(412 / 4760 ; 8.66 \%)$, women's care unit (249/4760; 5.23\%), coronary care unit $(128 / 4760 ; 2.69 \%)$, progressive care unit $(78 / 4760 ; 1.63 \%)$, and the labor, delivery, and recovery room $(43 / 4760 ; 0.90 \%)$. The specimen types collected consisted of: urine (2812/4760; $59.08 \%$ ), sputum $(561 / 4760 ; 11.79 \%)$, blood $(412 / 4760$; $8.66 \%)$, wound $(214 / 4760 ; 4.50 \%)$, vaginal $(212 / 4760$; $4.45 \%)$, throat $(135 / 4760 ; 2.84 \%)$, tracheal $(83 / 4760$; $1.74 \%)$, and other $(331 / 4760 ; 6.95 \%)$.

\section{Bacterial Identification and Antimicrobial Susceptibility Testing}

Isolated bacteria were streaked on plates of $5 \%$ sheep blood agar (Watin Biolife, Riyadh, K.S.A) and grown overnight at $37^{\circ} \mathrm{C}$ under $5 \% \mathrm{CO}_{2}$. Following incubation, one to three colonies were selected and used to inoculate $3 \mathrm{~mL}$ of $0.45 \%$ sterile sodium chloride solution, which was adjusted to a turbidity of $0.5 \mathrm{McF}$ arland. Bacterial suspensions were applied to each of two testing cards, VITEK 2 ID-GNB (an identification system) and ASTNo. 12 (a susceptibility testing system), loaded into a VITEK 2 system according to the manufacturer's instructions.

The VITEK 2 system is an automated instrument that uses fluorescence-based technology and is capable of rapid, simultaneous identification and antimicrobial sensitivity testing of bacteria consistent with the standards of the Clinical and Laboratory Standards Institute (CLSI). ${ }^{12,13}$ The system determines minimum inhibitory concentration (MIC) by comparing the growth of a patient isolate to that of isolates with known MICs. Therefore, the standard curve stored in the VITEK 2 relates organism activity in the antibiotic wells to reference MICs. The VITEK 2 system software (v. 8.01) then determines interpretive breakpoints using a guideline established by the CLSI. ${ }^{13}$ Consistent with CLSI recommendations, the following strains were also tested to ensure quality control: Escherichia coli ATCC 35,218, Pseudomonas aeruginosa ATCC 27,853, and K. pneumoniae ATCC 700,603. ${ }^{14}$

As defined by the US Centers for Disease Control and Prevention (CDC) and the European Centre for Disease Prevention and Control (ECDC), bacteria are classified as MDR if they have acquired non-susceptibility to at least one agent in each of three or more antibiotic categories. ${ }^{15}$ The susceptibility testing system used here included the following antibiotics and MIC reference ranges: amikacin (AK; MIC 2-64 $\mu \mathrm{g} / \mathrm{mL}$ ), amoxicillin/clavulanate (AMC; MIC 2/1-32/ $16 \mu \mathrm{g} / \mathrm{mL}$ ), ampicillin (AMP; MIC $2-32 \mu \mathrm{g} / \mathrm{mL}$ ), ceftazidime (CAZ; MIC 1-64 $\mu \mathrm{g} / \mathrm{mL}$ ), ciprofloxacin (CIP; MIC $0.25-4 \mu \mathrm{g} / \mathrm{mL}$ ), gentamicin (CN; MIC $1-16 \mu \mathrm{g} / \mathrm{mL}$ ), ceftriaxone (CRO; MIC 1-64 $\mu \mathrm{g} / \mathrm{mL}$ ), cefotaxime (CTX; MIC 1-64 $\mu \mathrm{g} / \mathrm{mL}$ ), cefuroxime (CXM; MIC 1-64 $\mu \mathrm{g} / \mathrm{mL}$ ), nitrofurantoin (F; MIC $0.5-16 \mu \mathrm{g} / \mathrm{mL}$ ), cefepime (FEP; MIC $1-64 \mu \mathrm{g} / \mathrm{mL}$ ), imipenem (IPM; MIC $0.25-12 \mu \mathrm{g} / \mathrm{mL}$ ), levofloxacin (LEV; MIC 0.12-8 $\mu \mathrm{g} / \mathrm{mL}$ ), meropenem (MEM; MIC $\quad 0.25-16 \mu \mathrm{g} / \mathrm{mL}$ ), trimethoprim/sulfamethoxazole (SXT; MIC 1/19-16/304 $\mu \mathrm{g} / \mathrm{mL}$ ), tigecycline (TGC; MIC 
0.5-8 $\mu \mathrm{g} / \mathrm{mL}$ ), and piperacillin/tazobactam (PTZ; MIC 4/4-128/4 $\mu \mathrm{g} / \mathrm{mL})$.

For ESBL detection, FEP $(1 \mu \mathrm{g} / \mathrm{mL})$, CTX $(0.5 \mu \mathrm{g} / \mathrm{mL})$, and CAZ $(0.5 \mu \mathrm{g} / \mathrm{mL})$ were used both alone and in combination with clavulanic acid $(10 \mu \mathrm{g} / \mathrm{mL}, 4 \mu \mathrm{g} / \mathrm{mL}$, or $4 \mu \mathrm{g} / \mathrm{mL}$, respectively), and isolates were classified as ESBL positive or negative according to the manufacturer's instructions.

\section{Statistical Analysis}

Data were extracted from the Laboratory Information System of the hospital and imported into spreadsheet software (Excel, Microsoft Corp.). For multiple comparisons between years, a mixed-model analysis was applied using Statistical Analysis System (SAS Institute, Inc.). For the adjustment of multiple comparisons, Tukey's method was performed, and $p$ values less than 0.05 were considered significant.

\section{Results}

\section{Reduction in Antibiotic Resistance of Isolated Gram-Negative Bacteria from 2017 to 2019}

A total of 4760 gram-negative bacterial isolates were collected over the three-year study period (Table 1). The most isolated organism was Escherichia coli, with 2585/ $4760(54.31 \%)$ strains, and the least isolated organism was Providencia stuartii, with 55/4760 (1.16\%) strains. Over the course of the study period, Escherichia coli exhibited reduced or equal resistance to most antibiotics with the exception of antibiotic F, for which resistance increased over time. The strongest AR reduction in Escherichia coli was observed for AK with -12\% (125/780, 16\%; 32/787, $4 \% ; 41 / 1018,4 \%$ for the years 2017, 2018, and 2019, respectively), followed by AMP with $-6 \%$ (515/780, $66 \% ; 480 / 787,61 \% ; 611 / 1018,60 \%)$ and CXM with $-6 \%$ (281/780, 36\%; 244/787, 31\%; 305/1018, 30\%).

For K. pneumoniae and Pseudomonas aeruginosa, the total number of AR isolates was $849 / 4760$ (17.84\%) and $482 / 4760(10.13 \%)$, respectively. Both of these species showed a reduction of resistance to all antibiotics between 2017 and 2019, with the exception of antibiotic $F$ in $K$. pneumoniae. For $K$. pneumoniae, the most profound reduction was in treatment with PTZ at $-28 \%(97 / 241$, $40 \%$; 96/310, 31\%; 36/298, 12\% for the years 2017, 2018, and 2019, respectively; $p<0.05$ ), while for Pseudomonas aeruginosa, the greatest reduction was for MEM with $-23 \%$ $(73 / 186,39 \% ; 31 / 155,20 \% ; 23 / 141,16 \% ; p<0.05)$.
For Proteus and Acinetobacter species, the total number of AR strains was 254/4760 (5.34\%) and 251/4760 (5.27\%), respectively. Both demonstrated reduced resistance to all antibiotics over the study period, except for SXT with Acinetobacter species. For Proteus species, the strongest reduction was observed with LEV at $-46 \%$ (70/107, 65\%; $35 / 84,42 \% ; 12 / 63,19 \%$ for the years 2017,2018 , and 2019 , respectively; $p<0.05$ ), while in Acinetobacter species, AK had the most reduction at $-40 \%(78 / 10276 \% ; 27 / 66,41 \%$; 30/83, 36\%; $p<0.05)$.

For Enterobacter cloacae and Citrobacter species, the total number of strains isolated with AR was 128/4760 (2.69\%) and 83/4760 (1.74\%), respectively. In Enterobacter cloacae, reduction in resistance was found for only three antibiotics (CTX, F, and PTZ), while Citrobacter species displayed reduced resistance over time for all antibiotics except CIP, IPM, MEM, and SXT.

For Salmonella species and Providencia stuartii, the number of isolated strains with AR was 73/4760 (1.53\%) and 55/4760 (1.16\%), respectively. Unfortunately, Salmonella species demonstrated increased resistance to all antibiotics over time, with AMP being the most impacted. However, Providencia stuartii displayed decreased resistance to all antibiotics over the study period, with CTX, CAZ, and FEP displaying the strongest reductions.

\section{Reduction in MDR and ESBL of Isolated Gram-Negative Bacteria Over Time}

The total number of gram-negative isolates with MDR was $851 / 4760$ (17.88\%). The most frequently isolated MDR bacteria was Escherichia coli (292/851; 34.31\%), followed by $K$. pneumoniae $(218 / 851 ; 25.62 \%)$, and Acinetobacter species $(167 / 851 ; 19.62 \%)$.

Generally, the proportion of MDR bacteria among AR isolates from a given genus decreased significantly over time $(p<0.05)$; the exceptions were Enterobacter cloacae and Citrobacter species (Figure 1). For example, Escherichia coli demonstrated MDR rates of 14.87\% (116/780), 9.78\% (77/787), and 9.72\% (99/1018) for the years 2017, 2018, and 2019 , respectively, giving a total decrease of $-5.15 \%$. The strongest reduction in MDR rate was displayed by K. pneumoniae with $-20.84 \%$ (85/241, 35.27\%; 90/310, $29.03 \%$; 43/298, 14.43\%).

For ESBL-positive clinical isolates, 1328/4760 $(27.90 \%)$ were obtained in total (Figure 2). The species most commonly ESBL-positive were Escherichia coli 


\begin{tabular}{|c|c|c|c|c|c|c|}
\hline E & 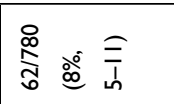 & 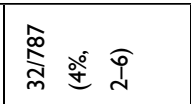 & 的 응 웅 & 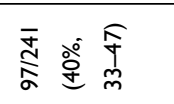 & 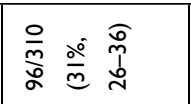 & 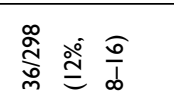 \\
\hline 斊 & 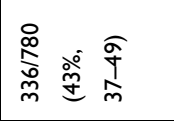 & 畣 & 类 & 亲遖 & 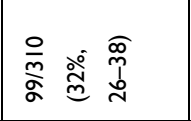 & 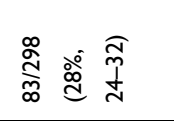 \\
\hline$\frac{\Sigma}{\Sigma}$ & 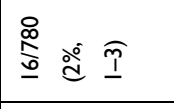 & 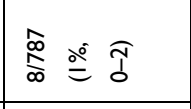 & 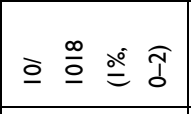 & 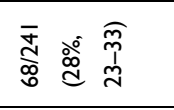 & 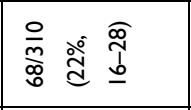 & 恋 \\
\hline 己̈ & 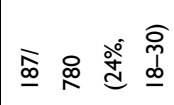 & 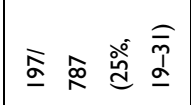 & 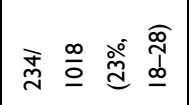 & 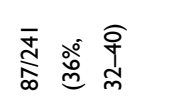 & 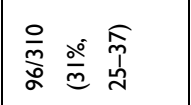 & 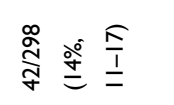 \\
\hline$\underline{\underline{\underline{\Sigma}}}$ & 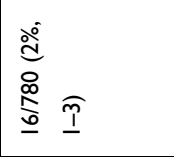 & 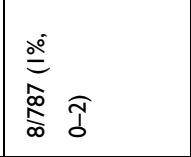 & 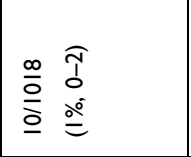 & 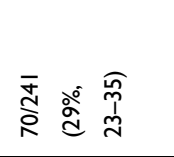 & 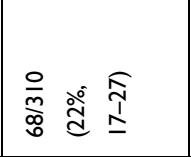 & 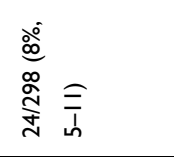 \\
\hline 总 & 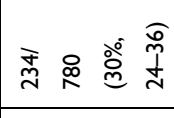 & 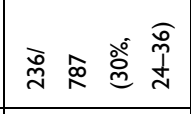 & 总 & 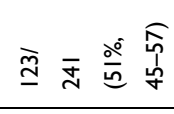 & 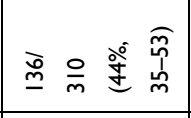 & $\begin{array}{l}\infty \\
\stackrel{\infty}{\tilde{N}} \underset{\infty}{\infty} \\
\stackrel{0}{\infty}\end{array}$ \\
\hline 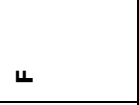 & 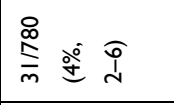 & 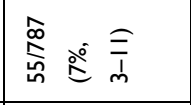 & 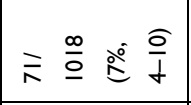 & 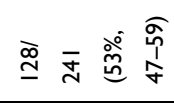 & 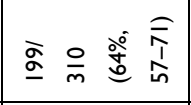 & مَ \\
\hline$\underbrace{\Sigma}_{0}$ & 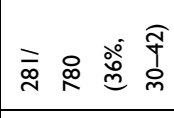 & 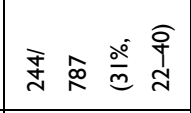 & 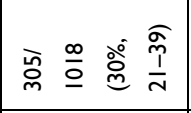 & 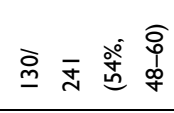 & 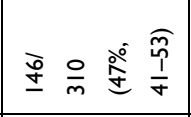 & 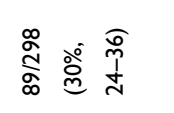 \\
\hline$\stackrel{x}{u}$ & 高造 商帝 & 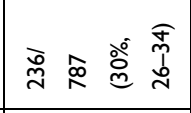 & 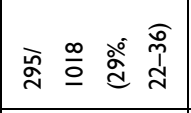 & 高无商旁 & 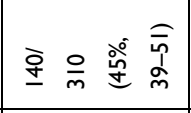 & 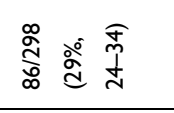 \\
\hline$\stackrel{\circ}{\check{U}}$ & 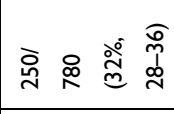 & 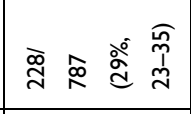 & 总 & 焉无商旁 & 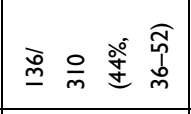 & 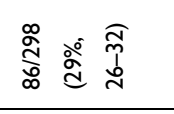 \\
\hline z & 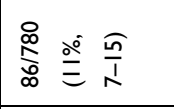 & 商 & 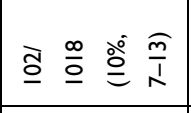 & 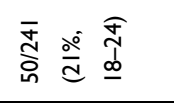 & 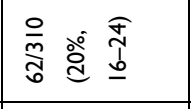 & 文 \\
\hline$\overline{\bar{u}}$ & 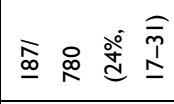 & 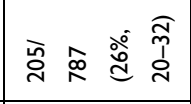 & 封 & 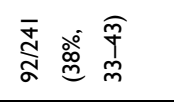 & 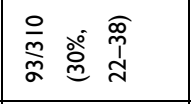 & 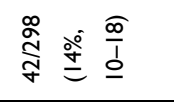 \\
\hline है & 高品 商悀 & 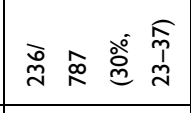 & 홍 & 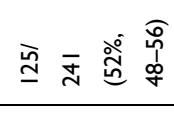 & 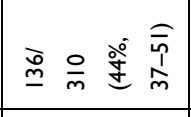 & 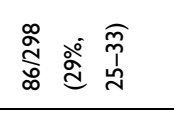 \\
\hline$\frac{0}{\frac{2}{4}}$ & 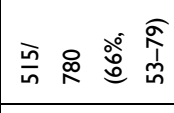 & 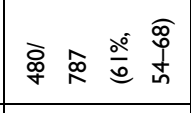 & 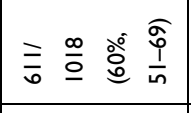 & & & \\
\hline$\frac{U}{4}$ & 高 & 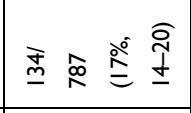 & 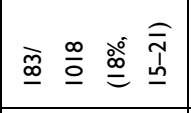 & 亲奇高 & 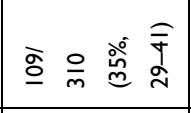 & 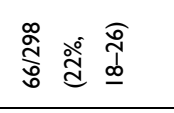 \\
\hline$\check{4}$ & 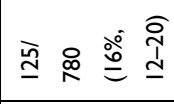 & 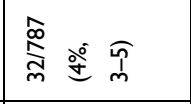 & 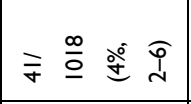 & 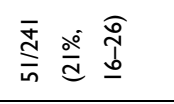 & 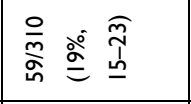 & 恋 \\
\hline 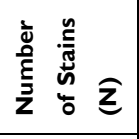 & $\stackrel{\infty}{\infty}$ & $\stackrel{\infty}{\infty}$ & $\stackrel{\infty}{\underline{0}}$ & $\bar{\beth}$ & $\stackrel{\circ}{m}$ & $\stackrel{\infty}{\text { ลั }}$ \\
\hline ટ્ઁ & $\overline{\mathrm{i}}$ & $\frac{\infty}{2}$ & $\frac{9}{9}$ & ì & $\frac{\infty}{2}$ & 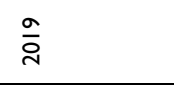 \\
\hline 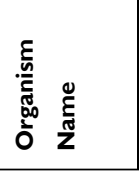 & \multicolumn{3}{|c|}{ 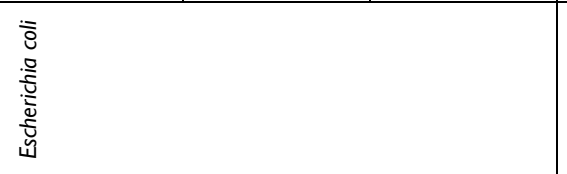 } & \multicolumn{3}{|l|}{ 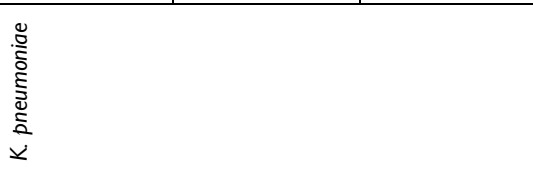 } \\
\hline
\end{tabular}




\begin{tabular}{|c|c|c|c|c|c|c|c|c|}
\hline 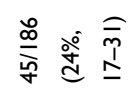 & 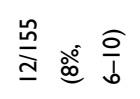 & 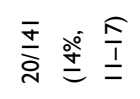 & 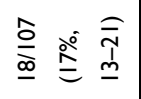 & 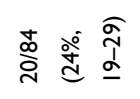 & $\stackrel{m}{\circ} \stackrel{\circ}{\circ} \stackrel{\frac{\sigma}{0}}{\frac{\sigma}{b}}$ & 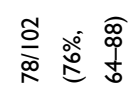 & 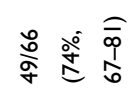 & 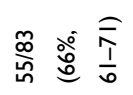 \\
\hline & & & 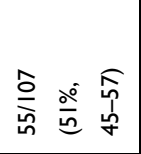 & 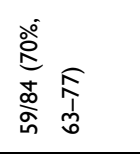 & 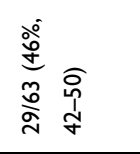 & 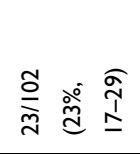 & 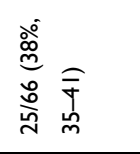 & 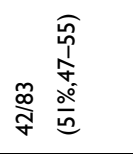 \\
\hline 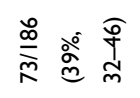 & 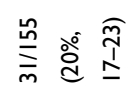 & 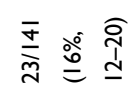 & 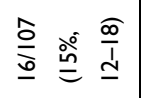 & 志 dơ & mo & 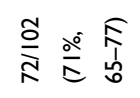 & 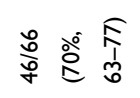 & 总 \\
\hline 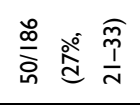 & 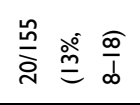 & 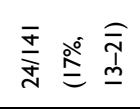 & 을 商 & 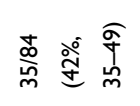 & 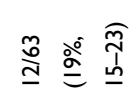 & 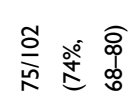 & 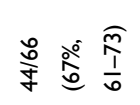 & 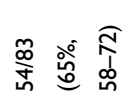 \\
\hline 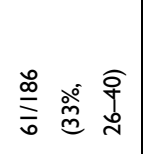 & 㞮 & 辤 & & & & 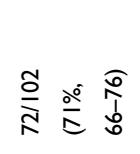 & 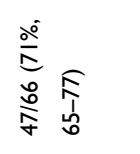 & 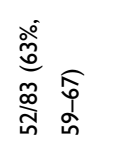 \\
\hline 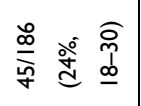 & 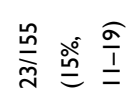 & 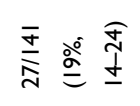 & 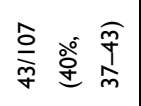 & 志 ơ & 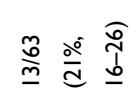 & 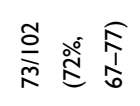 & 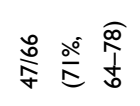 & 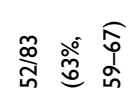 \\
\hline & & & 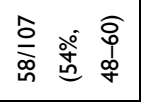 & 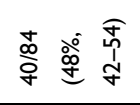 & mo & & & \\
\hline & & & 产高票亭 & 志兽 & 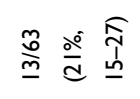 & & & \\
\hline & & & 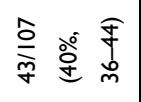 & 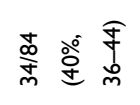 & 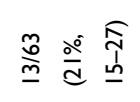 & & & \\
\hline 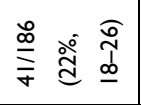 & 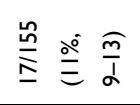 & 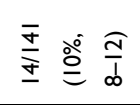 & 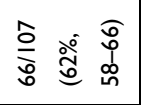 & 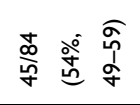 & 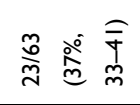 & 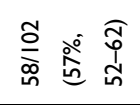 & 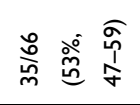 & 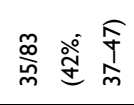 \\
\hline 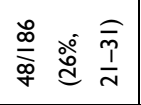 & 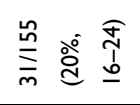 & 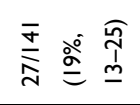 & 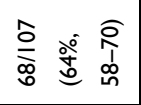 & 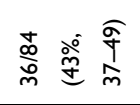 & 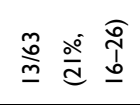 & 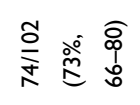 & 웅 & 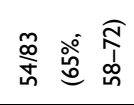 \\
\hline 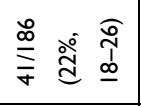 & 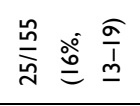 & 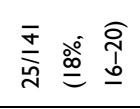 & 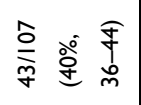 & 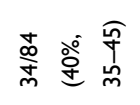 & 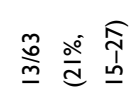 & 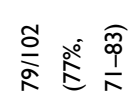 & 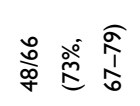 & 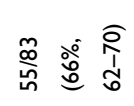 \\
\hline & & & 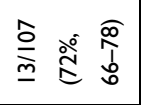 & 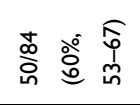 & 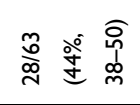 & & & \\
\hline & & & 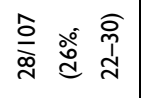 & 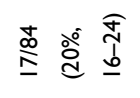 & ๕̊ㅇㅇ & & & \\
\hline 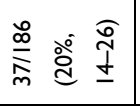 & $\stackrel{\stackrel{n}{\frac{n}{6}} \stackrel{\circ}{\circ} \frac{0}{b}}{b}$ & $\stackrel{\bar{\sigma}}{\underline{\infty} \stackrel{\circ}{\stackrel{\delta}{\rho}} \frac{\kappa}{\sigma}}$ & 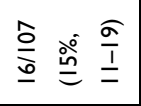 & 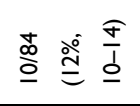 & 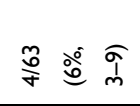 & 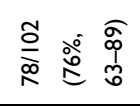 & 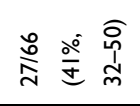 & 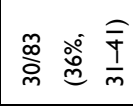 \\
\hline$\stackrel{\circ}{\infty}$ & 늠 & $\bar{\Xi}$ & $\underline{\underline{o}}$ & \$ & 3 & $\underline{\widetilde{s}}$ & $\stackrel{\circ}{\circ}$ & 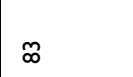 \\
\hline$\overline{\bar{N}}$ & $\frac{\infty}{i}$ & ì & $\hat{\bar{i}}$ & $\frac{\infty}{2}$ & $\frac{9}{2}$ & ลे & $\frac{\infty}{2}$ & $\frac{9}{2}$ \\
\hline \multicolumn{3}{|l|}{ 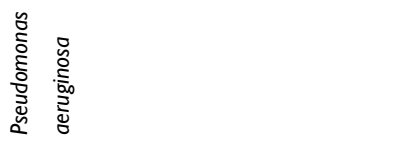 } & \multicolumn{3}{|l|}{ 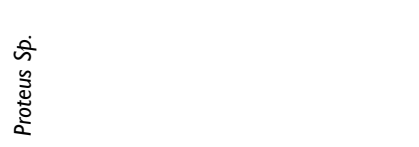 } & \multicolumn{3}{|l|}{ 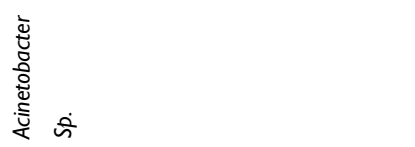 } \\
\hline
\end{tabular}




\begin{tabular}{|c|c|c|c|c|c|c|c|c|c|}
\hline$\stackrel{2}{2}$ & 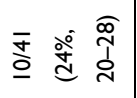 & 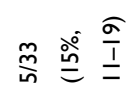 & 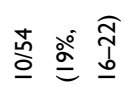 & ลิ 尺ें & సิ ब̊ㅇㅇ & 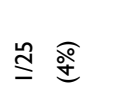 & & & \\
\hline 爻 & 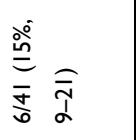 & 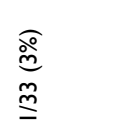 & 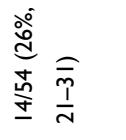 & $\begin{array}{l}\text { ळ. } \\
\text { ले } \\
\text { Iิ }\end{array}$ & 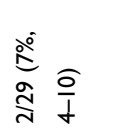 & 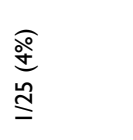 & 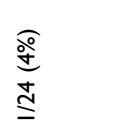 & 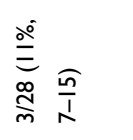 & 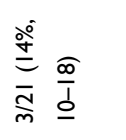 \\
\hline 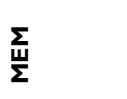 & $\Xi \stackrel{\Xi}{\stackrel{\rho}{I}}$ & 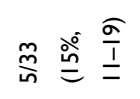 & 㟥落 & ฐิ ळ̊ & ฐิ ळ̊ & $\stackrel{\text { ఏ }}{=}$ & & & \\
\hline 㞼 & F & $\stackrel{m}{\circ} \stackrel{\circ}{\stackrel{\circ}{c}}$ & 䓂高定 & ฐิ & 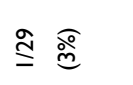 & 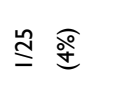 & & & \\
\hline$\sum_{\underline{\underline{a}}}$ & 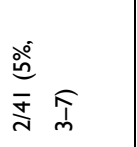 & 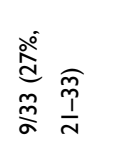 & 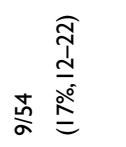 & $\begin{array}{l}\text { ळे } \\
\text { ปे }\end{array}$ & $\begin{array}{l}\text { ळे } \\
\text { ড̀ }\end{array}$ & 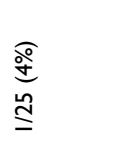 & & & \\
\hline 这 & 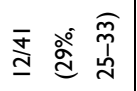 & 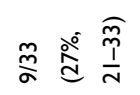 & 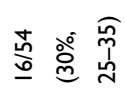 & 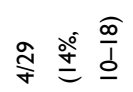 & 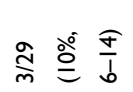 & 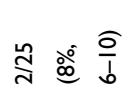 & & & \\
\hline ц & 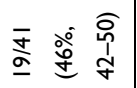 & 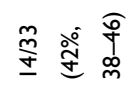 & 壱 学 & 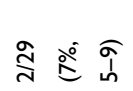 & 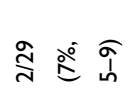 & 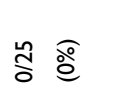 & & & \\
\hline 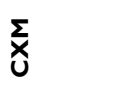 & & & & 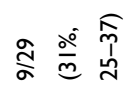 & 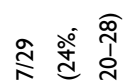 & 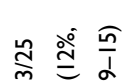 & & & \\
\hline$\stackrel{x}{u}$ & 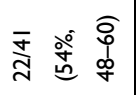 & m. & 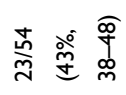 & 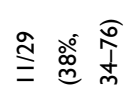 & 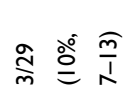 & 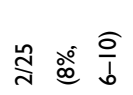 & & & \\
\hline Ů & 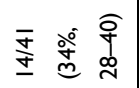 & 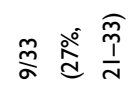 & 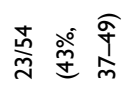 & 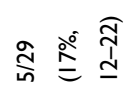 & 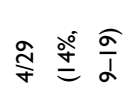 & 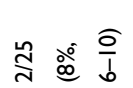 & & & \\
\hline z & 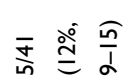 & 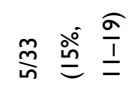 & 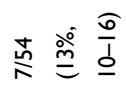 & సิ ळ̊ㅇㅇ & 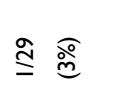 & 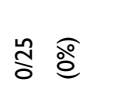 & & & \\
\hline $\bar{u}$ & 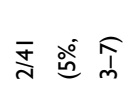 & 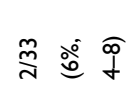 & 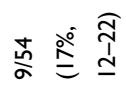 & 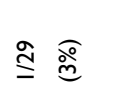 & 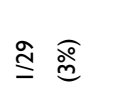 & 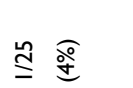 & 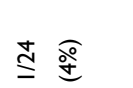 & 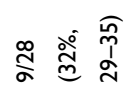 & 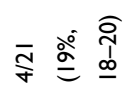 \\
\hline Ư & 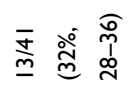 & 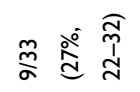 & 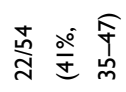 & ๙ิ & $\stackrel{\text { sิ }}{\stackrel{0}{\circ}}$ & 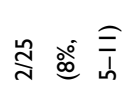 & & & \\
\hline$\frac{0}{\varepsilon}$ & & & & & & & 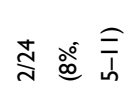 & $\stackrel{\infty}{m} \stackrel{\circ}{\stackrel{0}{\varrho}} \frac{\widehat{n}}{\Gamma}$ & 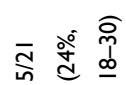 \\
\hline$\frac{u}{\alpha}$ & & & & 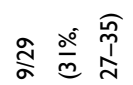 & 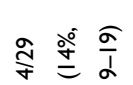 & 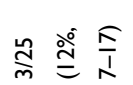 & & & \\
\hline$\check{\alpha}$ & ‡ & $\stackrel{m}{\circ}$ ஓ̊ & 䓂 & ฐิ ळ̊ & ฐิ & $\stackrel{\check{\Xi}}{\mathrm{o}}$ & & & \\
\hline 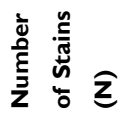 & $\bar{F}$ & $\stackrel{m}{m}$ & 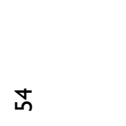 & নे & సे & $\stackrel{\sim}{\sim}$ & $\stackrel{\searrow}{\sim}$ & $\stackrel{\infty}{\sim}$ & $\bar{N}$ \\
\hline 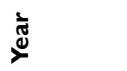 & ì & $\frac{\infty}{2}$ & $\frac{9}{2}$ & 혼 & $\frac{\infty}{a}$ & $\frac{9}{2}$ & 숭 & $\frac{\infty}{a}$ & $\frac{0}{2}$ \\
\hline 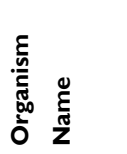 & \multicolumn{3}{|l|}{ 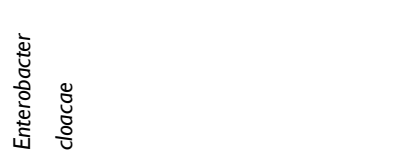 } & \multicolumn{3}{|l|}{ 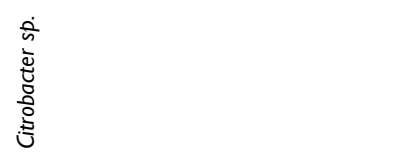 } & \multicolumn{3}{|l|}{ 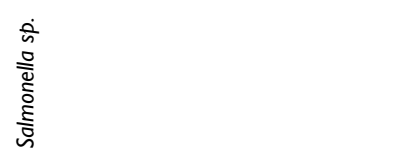 } \\
\hline
\end{tabular}




\begin{tabular}{|c|c|c|c|}
\hline 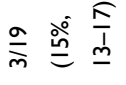 & 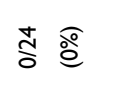 & $\grave{\partial} \stackrel{\circ}{\stackrel{8}{\circ}}$ & \\
\hline 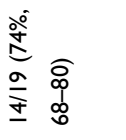 & 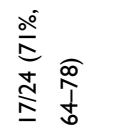 & 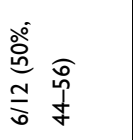 & \\
\hline$\stackrel{\circ}{\equiv}$ 。ํํㅇ & 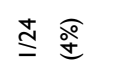 & 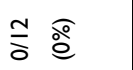 & \\
\hline 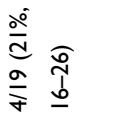 & 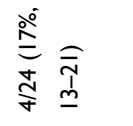 & 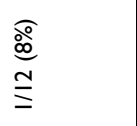 & \\
\hline 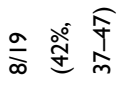 & 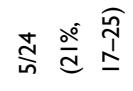 & $\check{亏} \widehat{\circ}$ & \\
\hline 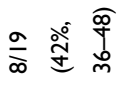 & 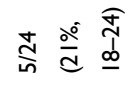 & 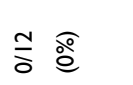 & \\
\hline 咅守 & 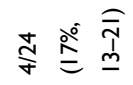 & 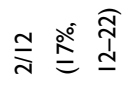 & \\
\hline 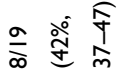 & 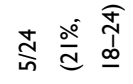 & $\frac{\cong}{\partial} \stackrel{\circ}{g}$ & \\
\hline 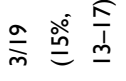 & 志官高 & $\stackrel{\circ}{\cong}$ & \\
\hline$\underline{\underline{a}}$ & $\stackrel{\sim}{*}$ & $\simeq$ & $\begin{array}{l}0 \\
\stackrel{0}{f} \\
\stackrel{f}{2}\end{array}$ \\
\hline ì & $\frac{\infty}{\grave{d}}$ & $\frac{\circ}{2}$ & \\
\hline 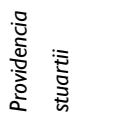 & & & $\begin{array}{l}\overline{\mathrm{J}} \\
\stackrel{5}{\circ}\end{array}$ \\
\hline
\end{tabular}

(772/1328; 58.13\%) and K. pneumoniae (345/1328; 25.98\%). The proportion of ESBL-positive Escherichia coli decreased over the study period, with values of $31.79 \%$ (248/780), 29.61\% (233/787), and 28.59\% (291/ 1018 ) for the years 2017,2018 , and 2019 , respectively. Out of all ESBL-positive bacteria identified, the strongest rate reduction was displayed by Providencia stuartii with $-33.78 \%$ (8/19, 42.11\%; 51/240, 21.25\%; 1/12, 8.33\%), followed by $K$. pneumoniae with $-23.68 \%$ (125/241, $51.87 \% ; 136 / 310,43.87 \% ; 84 / 298,28.19 \%)$. In addition, our data demonstrated significant reductions $(p<0.05)$ in ESBL rate over time for K. pneumoniae, Proteus species, Citrobacter species, and Providencia stuartii.

\section{Discussion}

The prevalence of AR and the distribution of resistant agents have become a major concern for physicians and scientists. ${ }^{3}$ Unfortunately, several inappropriate practices that contribute to AR are known to occur in Saudi, including buying antibiotic drugs without prescription, self-medication with antibiotics, and failure to complete prescribed antibiotic regimens. ${ }^{16,17}$ In 2014 , the reported prevalence of selfmedication with antibiotics was $78.7 \%,{ }^{17}$ while in 2015 , approximately $64 \%$ of people in Saudi used antibiotics without prescription, and $71 \%$ did not finish a prescribed antibiotic treatment. ${ }^{16}$ In addition, the lack of AR surveillance programs led to increased inappropriate use of antibiotics among health care staff and patients. ${ }^{18}$ Over time, these inappropriate practices can allow bacteria to share $A R$ genes and factors such as biofilm production components that enhance resistance toward antibiotics, which can increase the prevalence of ESBL and MDR, thereby leading to more complicated infections. ${ }^{19-21}$ Furthermore, several factors have caused the development of new antibiotic drugs to slow, and methods for overcoming AR are limited. Bacterial pathogens resistant to ordinary antibiotics require combination therapy, in which two or more antibiotics are used to enhance the efficacy of the drugs. ${ }^{22,23}$ Increased prevalence of antibiotic resistance therefore presents difficulties for physicians and constitutes a growing threat to public health.

Accordingly, in 2017, the Saudi Ministry of Health implemented several strategies to reduce the risk of AR, including raising national awareness and improving knowledge by establishing evidence-based communication, training, and development programs; these programs targeted the public along with both human and animal healthcare providers. In addition, the Ministry established 


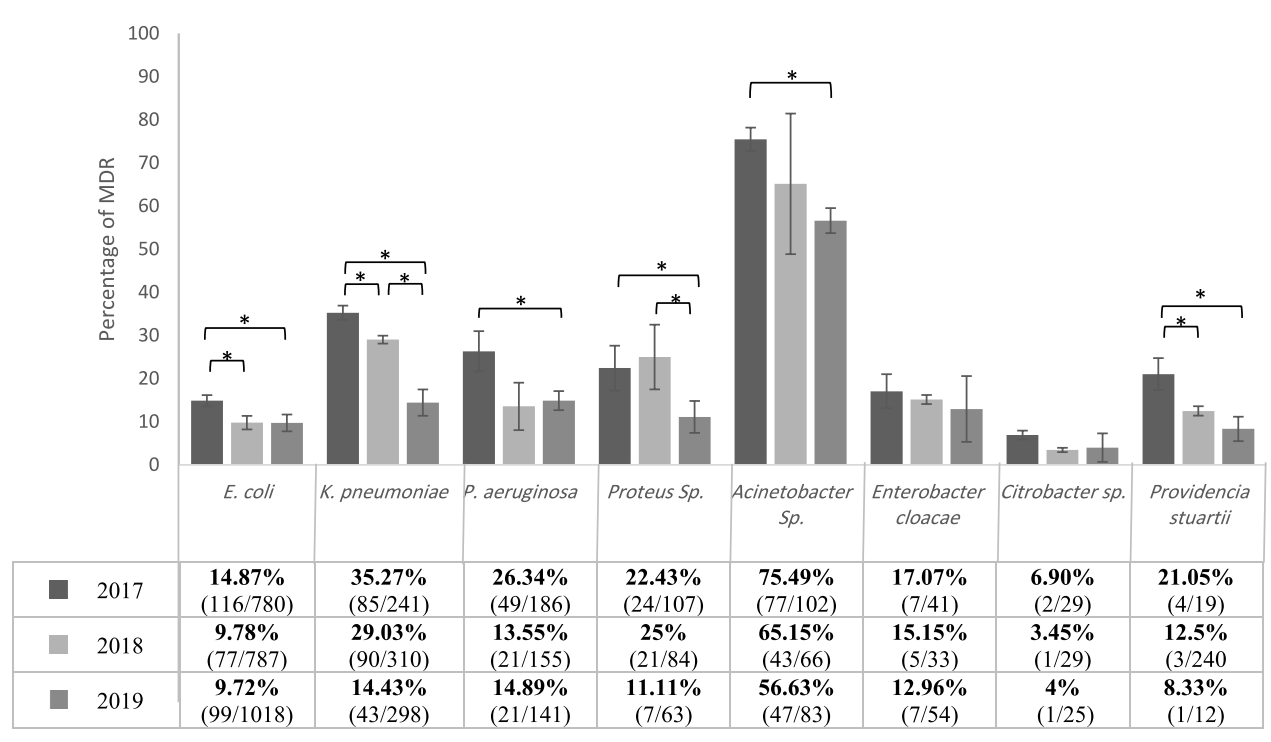

Figure I Three years evaluation of MDR of isolated gram-negative bacteria. Results were presented as percentage \pm SD of MDR of isolated bacteria compared to their antibiotic resistance. Mixed-model analysis was performed for the multiple comparisons, and *Indicates a significant difference in multi-drug resistance between years $(p<0.05)$.

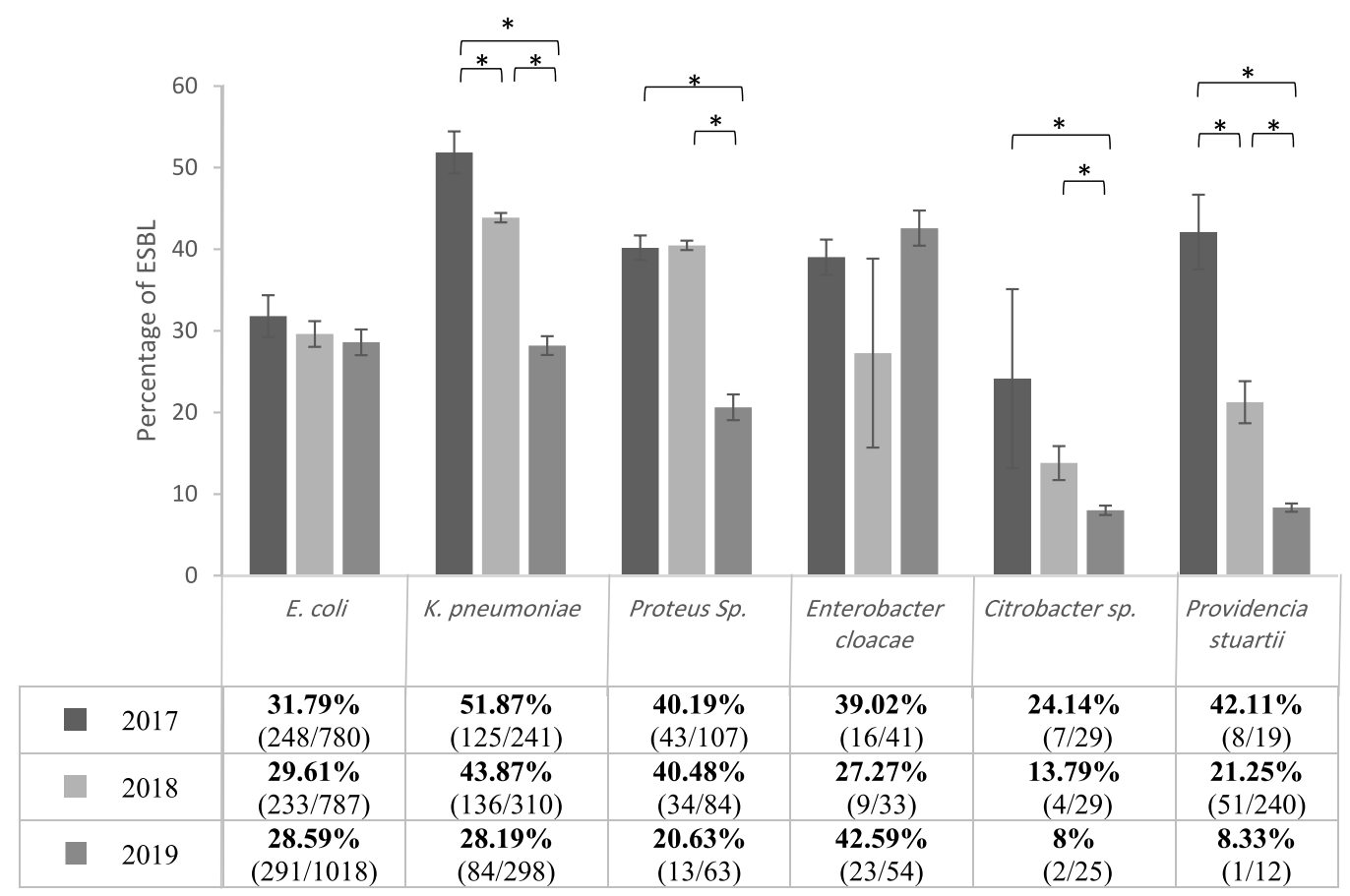

Figure 2 Three years evaluation of ESBL of isolated gram-negative bacteria. Results were presented as percentage $\pm S D$ of ESBL of isolated bacteria compared to their antibiotic resistance strain. Mixed-model analysis was performed for the multiple comparisons, and *Indicates a significant difference in ESBL between years ( $p<0.05$ ).

a national surveillance system for AR, designating seven reference microbiology laboratory facilities (College of American Pathologists accredited), and 12 human and six animal AR surveillance sentinel sites in different regions of Saudi Arabia; all sentinel sites are connected to the national center for analysis and reporting. The Ministry also set out to decrease the incidence of infection by introducing an infection prevention and control program in veterinary settings and animal husbandry contexts, and by applying infection prevention and control guidelines to limit the spread of AR outside health settings. Moreover, the Ministry aimed to optimize the use of antimicrobial medicines in human and animal health by strengthening the pharmaceutical supply chain, ensuring uninterrupted 
access to high-quality antimicrobial medicines, and by monitoring for and improving the appropriate use of antimicrobial agents in health care. ${ }^{9,10}$

In this study, we analyzed the prevalence of AR, ESBL, and MDR among key pathogenic gram-negative bacterial species isolated from patients in a private hospital in Saudi Arabia. From January 2017 to December 2019, we collected a total of 4760 gram-negative bacterial isolates. The most isolated organism was Escherichia coli, with $2585(54.30 \%)$ strains, this finding is in agreement with a study conducted by Buetti and colleagues. ${ }^{24}$ However, in other studies by Sedigh et al and Alam et al, the most isolated gram-negative AR bacteria were Acinetobacter species. ${ }^{25,26}$

We observed that resistance to most antibiotics reduced over time in all studied bacteria except Salmonella species, which is in agreement with studies done by Mukherjee et al and Browne et al. ${ }^{28,29}$ For Escherichia coli, the greatest reduction was with the antibiotic AK $(-12 \%)$, while for $K$. pneumoniae the strongest reduction was for PTZ $(-28 \%)$, and in Pseudomonas aeruginosa for MEM $(-23 \%)$. In contrast, in Iran, a country with no systematic guidelines for antibiotic usage, Azimi et al showed that resistance to most antibiotics increased continuously from 2016 to 2018; in particular, they reported increased resistance of Escherichia coli to AK (60\%), K. pneumoniae to PTZ (24\%), and Pseudomonas aeruginosa to MEM $(7 \%){ }^{27}$ The contrast between their findings and the present study emphasizes the import of AR monitoring. Interestingly, our data displayed that some bacterial strains developed complete susceptibility to an antibiotic over time, such as for Proteus species treated with AMC (28/ $107,26 \% ; 17 / 84,20 \% ; 0 / 63,0 \%$ for the years 2017,2018 , and 2019, respectively) and Providencia stuartii treated with CTX (8/19, 42\%; 5/24, 22\%; 0/12, 0\%).

Taken together, these results indicate that AR monitoring is important and has several benefits, such as providing data on bacterial resistance frequency, assisting in the selection of ideal antibiotics and subsequently reducing AR prevalence, decreasing hospitalization rate and treatment costs, and reducing the death rate. ${ }^{27}$ As resistance in Salmonella species was reported to be more persistent, ${ }^{28,29}$ the SNAP has specifically implemented surveillance for the detection of Salmonella species and total bacterial count in poultry in all regions of the Saudi Kingdom, and restricts the use of antibiotics critically important for human medicine in food production animals. ${ }^{10}$ Notably, our results do not reflect the effect of these two implemented strategies, as more time is needed to analyze their consequences.

In the present study, Escherichia coli and K. pneumoniae together comprised $84 \%$ of ESBL-positive isolates and $60 \%$ of MDR isolates. Similarly, Hayati et al and Deng et al demonstrated that ESBL activity and MDR were most frequent in Escherichia coli and K. pneumoniae, at a combined $83 \%$ and $51 \%$, respectively. ${ }^{30,31}$ In March 2017, the Saudi Health Ministry adapted and applied the WHO global antimicrobial resistance surveillance system (GLASS) with priority for common ESBL and MDR bacteria, including Escherichia coli and $K$. pneumoniae. In addition, in April 2017, the Health Ministry requested a stewardship program key performance indicator on the process and outcome regarding ESBL and MDR bacteria. ${ }^{10}$ Our data showed that after implementing these strategies, in addition to a generally noticeable decrease of all MDR and ESBL bacteria from 2017 to 2019, the prevalence of ESBL and MDR Escherichia coli isolates decreased slightly, while for $K$. pneumoniae the prevalence decreased significantly $(p<0.05)$. The reductions in ESBL and MDR had fortunate consequences such as raising the rate of treatment success and decreasing rates of morbidity and mortality. ${ }^{6,32-34}$

While we found resistance to all antibiotics to generally reduce over time, SXT was an exception. This finding is probably linked to patterns in the use of antimicrobial therapy and may reflect selective reporting of resistance testing. ${ }^{35}$ In terms of general trends in resistance over the study period, AMP was observed to have the highest resistance rate, while AK had the lowest. Extremely high resistance of gram-negative pathogens to AMP was also reported in Saudi by Al-Tawfiq et al and in India by Dharmapalan et al. ${ }^{36,37}$ Interestingly, we found the AMP resistance rate to decrease over time; meanwhile, in Iran, which had no systematic guidelines for antibiotic usage, Azimi et al documented an increase in AMP resistance rate. $^{27}$

It should be noted that our study has several limitations, including 1) clinical data regarding treatment outcomes and mortality rate due to gram-negative bacteria were not obtainable; thus, we could not consider this information in our analysis; 2) control groups for some variables were not included; 3) identification of Proteus, Acinetobacter, Citrobacter, and Salmonella was not done to species level; thus, it is impossible to determine the AR and infection rates for particular species of these bacteria; 4) the analysis did not consider annual changes in hospital policies due to physician recommendations and 
availability of antibiotics; 5) gram-positive bacteria were not included; 6) while we used quality control strains for the VITEK system, the study did not include an ARnegative control group; and 7) the total number of tested isolates differed from year to year.

Despite these limitations, our results clearly show that the strategies implemented by the SNAP are effective in reducing AR. To our knowledge, this study is the first to investigate the efficacy of the SNAP since its implementation in 2017. It would be interesting to continue with future studies that increase the number of hospitals and years considered to gain a broader overview of the implemented plan's success.

\section{Conclusion}

The results of this study suggest that the SNAP is effective in reducing the rates of $\mathrm{AR}, \mathrm{ESBL}$, and $\mathrm{MDR}$ in most gram-negative bacteria. Moreover, we have demonstrated that from 2017 to 2019, some bacterial strains developed complete susceptibility to a particular antibiotic, such as Proteus species treated with AMC and Providencia stuartii treated with CTX. However, Salmonella species showed increased resistance to all antibiotics over time, and further investigation is required to improve the effectiveness of the implemented strategies. In addition, more studies including gram-positive bacteria merit consideration to gain a more complete perspective on the success of SNAP.

\section{Ethics Statement}

The study was approved by the Ethics Review Committee (ERC) of College of Medicine, Imam Mohammad Ibn Saud Islamic University. The ethics committee approved the waiver of patient's informed consent, with the justification that this was a retrospective and analytical study whose information was obtained from medical records. Also, the data were de-identified and anonymously analyzed. The study was conducted in accordance with the ethical guidelines of the Declaration of Helsinki. Privacy statement: the authors guarantee patient data confidentiality.

\section{Acknowledgments}

We are grateful to all hospital laboratory members for their support and give especial thanks to Meshari Almaliky for gathering and providing some missing data needed in our study. Also, we thank Imam Mohammad Ibn Saud Islamic University and SmartLab for supplying the funding.

\section{Author Contributions}

All authors made a significant contribution to the work reported, whether that is in the conception, study design, execution, acquisition of data, analysis and interpretation, or in all these areas; took part in drafting, revising or critically reviewing the article; gave final approval of the version to be published; have agreed on the journal to which the article has been submitted; and agree to be accountable for all aspects of the work.

\section{Funding}

This work was supported by Imam Muhammad Ibn Saud Islamic University and SmartLab.

\section{Disclosure}

The authors report no conflicts of interest for this work.

\section{References}

1. O'Brien TF, Clark A, Peters R, Stelling J. Why surveillance of antimicrobial resistance needs to be automated and comprehensive. $J$ Glob Antimicrob Resist. 2018;17:8-15. doi:10.1016/j.jgar.2018.10.011

2. Sharahi JY, Azimi T, Shariati A, Safari H, Tehrani MK, Hashemi A. Advanced strategies for combating bacterial biofilms. J Cell Physiol. 2019;234:14689-14708. doi:10.1002/jcp.28225

3. Ventola CL. The antibiotic resistance crisis: part1: causes and threats. Pharm Ther. 2015;40:277-283.

4. Fahrenkamp-Uppenbrink J. Countering antibiotic resistance. Science. 2015;347:1109-1111. doi:10.1126/science.347.6226.1109-q

5. O'Neil J Review on antimicrobial resistance. Antimicrobial resistance: tackling a crisis for the health and wealth of nations. London; review on antimicrobial resistance; 2014. Available from: https://amr-review.org/sites/default/files/AMR\%20Review\%20Paper $\% 20-\% 20$ Tackling $\% 20 \mathrm{a} \% 20$ crisis $\% 20$ for $\% 20$ the $\% 20$ health $\% 20$ and $\% 20$ wealth $\% 20$ of $\% 20$ nations_1.pdf. Accessed October 8, 2020.

6. Mahmoudi S, Mahzari M, Banar M, et al. Antimicrobial resistance patterns of Gram-negative bacteria isolated from bloodstream infections in an Iranian referral paediatric hospital: a 5.5-year study. $J$ Glob Antimicrob Resist. 2017;11:17-22. doi:10.1016/j.jgar.2017.04.013

7. World Health Organization. Global action plan on antimicrobial resistance. Available from: https://www.who.int/antimicrobialresistance/global-action-plan/en/. Accessed October 8, 2020.

8. World Health Organization. Global antimicrobial resistance surveillance system (GLASS) report: early implementation 2017-2018. Available from: https://www.who.int/glass/resources/publications/earlyimplementation-report-2017-2018/en/. Accessed October 8, 2020.

9. Saudi Ministry of Health. Who publishes the Saudi national action plan for combating antibiotic-resistant bacteria on its portal. Available from: https://www.moh.gov.sa/en/Ministry/MediaCenter/ News/Pages/News-2017-11-19-002.aspx. Accessed October 8, 2020.

10. Saudi Ministry of Health. Kingdom Saudi Arabia national action on plan on combating antimicrobial resistance. Available from: http:// extwprlegs1.fao.org/docs/pdf/sau171813.pdf. Accessed October 8, 2020.

11. National Guard Health Affairs. Infection prevention and control manual. Available from: https://www.moh.gov.sa/CCC/healthp/regula tions/Documents/GCC\%20Infection\%20control\%20manual\% 202013\%20revisedOPT.pdf. Accessed October 8, 2020.

12. Funke G, Monnet D, Bernardis C, et al. Evaluation of the VITEK 2 system for rapid identification of medically relevant gram-negative rods. J Clin Microbiol. 1998;36:1948-1952. doi:10.1128/JCM.36.7.19481952.1998 
13. Clinical and Laboratory Standards Institute. Performance standards for antimicrobial susceptibility testing; twenty fourth informational supplement. CLSI M100-S24. Available from: https://kaldur.landspi tali.is/focal/gaedahandbaekur/gnhsykla.nsf/5e $27 \mathrm{f} 2 \mathrm{e} 5 \mathrm{a} 88$ c898e00256500003c98c2/9c4f4955ccb9f8100025751a0046b075/ SFILE/ATTJRR31.pdf/M100-S24\%20Performance\%20Standards\% 20for\%20Antimicrobial\%20Susceptibility\%20Testing.pdf. Accessed October 8, 2020

14. Wang M, Wei H, Zhao Y, et al. Analysis of multidrug-resistant bacteria in 3223 patients with hospital-acquired infections (HAI) from a tertiary general hospital in China. Bosn J Basic Med Sci. 2019;19:86-93. doi:10.17305/bjbms.2018.3826

15. Magiorakos AP, Srinivasan A, Carey RB, et al. Multidrug resistant, extensively drug-resistant and pandrug-resistant bacteria: an international expert proposal for interim standard definitions for acquired resistance. Clin Microbiol Infect. 2012;18:268-281. doi:10.1111/ j.1469-0691.2011.03570.x

16. El Zowalaty ME, Belkina T, Bahashwan SA, et al. Knowledge, awareness, and attitudes toward antibiotic use and antimicrobial resistance among Saudi population. Int $J$ Clin Pharm. 2016;38:1261-1268. doi:10.1007/s11096-016-0362-x

17. Al Rasheed A, Yagoub U, Alkhashan H, et al. Prevalence and predictors of self-medication with antibiotics in Al Wazarat Health Center, Riyadh City, KSA. Biomed Res Int. 2016;12:1-8. doi:10.1155/2016/3916874

18. Prestinaci F, Pezzotti P, Pantosti A. Antimicrobial resistance: a global multifaceted phenomenon. Pathog Glob Health. 2015;109:309-318. doi:10.1179/2047773215Y.0000000030

19. Alabdullatif M, Ramirez-Arcos S. Biofilm-associated accumulation-associated protein (Aap): a contributing factor to the predominant growth of Staphylococcus epidermidis in platelet concentrates. Vox Sang. 2019;114:28-37. doi:10.1111/vox.12729

20. Singh S, Singh SK, Chowdhury I, Singh R. Understanding the mechanism of bacterial biofilms resistance to antimicrobial agents. Open Microbiol J. 2017;11:53-62. doi:10.2174/1874285801711010053

21. von Wintersdorff CJ, Penders J, van Niekerk JM, et al. Dissemination of antimicrobial resistance in microbial ecosystems through horizontal gene transfer. Front Microbiol. 2016;7:173. doi:10.3389/ fmicb.2016.00173

22. Laws MR, Shaaban A, Rahman K. Antibiotic resistance breakers: current approaches and future directions. FEMS Microbiol Rev. 2019;43:490-516.

23. Alabdullatif M, Atreya CD, Ramirez-Arcos S. Antimicrobial peptides: an effective approach to prevent bacterial biofilm formation in platelet concentrates. Transfusion. 2018;58:2013-2021. doi:10. $1111 /$ trf. 14646

24. Buetti N, Atkinson A, Kottanattu L, et al. Patterns and trends of pediatric bloodstream infections: a 7-year surveillance study. Eur J Clin Microbiol Infect Dis. 2017;36:537-544. doi:10.1007/s10096-016-2830-6

25. Sedigh EH, Motamedifar M, Mansury D, et al. Bacterial etiology and antibacterial susceptibility patterns of pediatric bloodstream infections: a two year study from Nemazee Hospital, Shiraz, Iran J Compr Pediatr. 2016;7:e29929.

Infection and Drug Resistance

\section{Publish your work in this journal}

Infection and Drug Resistance is an international, peer-reviewed openaccess journal that focuses on the optimal treatment of infection (bacterial, fungal and viral) and the development and institution of preventive strategies to minimize the development and spread of resistance. The journal is specifically concerned with the epidemiology of
26. Alam MS, Pillai PK, Kapur P, Pillai KK. Resistant patterns of bacteria isolated from bloodstream infections at a university hospital in Delhi. J Pharm Bioallied Sci. 2011;3:525-530. doi:10.4103/0975-7406.90106

27. Azimi T, Maham S, Fallah F, et al. Evaluating the antimicrobial resistance patterns among major bacterial pathogens isolated from clinical specimens taken from patients in Mofid Children's Hospital, Tehran, Iran: 2013-2018. Infect Drug Resist. 2019;12:2089-2102. doi:10.2147/IDR.S215329

28. Mukherjee S, Anderson CM, Mosci RE, et al. Increasing frequencies of antibiotic resistant non-typhoidal Salmonella infections in Michigan and risk factors for disease. Front Med (Lausanne). 2019;6:250. doi:10.3389/fmed.2019.00250

29. Browne AJ, Kashef Hamadani BH, Kumaran EAP, et al. Drug-resistant enteric fever worldwide, 1990 to 2018: a systematic review and meta-analysis. BMC Med. 2020;18:1. doi:10.1186/s12916-019-1443-1

30. Deng Q, Li Q, Lin XM, Li YM. Epidemiology and antimicrobial resistance of clinical isolates about hospital infection from patients with hematological disease. Zhonghua Xие Yе Xие Za Zhi. 2012;33:994-999.

31. Hayati Z, Rizal S, Putri R. Isolation of extended-spectrum B-lactamase (ESBL) producing Escherichia coli and Klebsiella pneumoniae from Dr. Zainoel Abidin General Hospital, Aceh. Int J Trop Vet Biomed Res. 2019;4:16-22. doi:10.21157/ijtvbr.v4i1.13806

32. Gur D, Gulay Z, Akan OA, et al. Resistance to newer beta-lactams and related ESBL types in gram-negative nosocomial isolates in Turkish hospitals: results of the multicenter HITIT study. Mikrobiyol Bul. 2008;42:537-544.

33. Founou RC, Founou LL, Essack SY, Butaye P. Clinical and economic impact of antibiotic resistance in developing countries: a systematic review and meta-analysis. PLoS One. 2017;12:e0189621. doi:10.1371/journal.pone.0189621

34. Peters L, Olson L, Khu DTK, et al. Multiple antibiotic resistance as a risk factor for mortality and prolonged hospital stay: a cohort study among neonatal intensive care patients with hospital-acquired infections caused by gram-negative bacteria in Vietnam. PLoS One. 2019;14:e0215666. doi:10.1371/journal.pone.0215666

35. Al-Tawfiq JA, Momattin H, Al-Habboubi F, Dancer SJ. Restrictive reporting of selected antimicrobial susceptibilities influences clinical prescribing. J Infect Public Health. 2015;8:234-241. doi:10.1016/j. jiph.2014.09.004

36. Al-Tawfiq JA, Rabaan AA, Saunar JV, Bazzi AM. Antimicrobial resistance of gram-negative bacteria: a six-year longitudinal study in a hospital in Saudi Arabia. J Infect Public Health. 2020;13:737-745. doi:10.1016/j.jiph.2020.01.004

37. Dharmapalan D, Shet A, Yewale V, Sharland M. High reported rates of antimicrobial resistance in Indian neonatal and pediatric blood stream infections. J Pediatric Infect Dis Soc. 2017;6:e62-e68. doi:10.1093/jpids/piw092 antibiotic resistance and the mechanisms of resistance development and diffusion in both hospitals and the community. The manuscript management system is completely online and includes a very quick and fair peerreview system, which is all easy to use. Visit http://www.dovepress.com/ testimonials.php to read real quotes from published authors. 